\title{
Removing Barriers: On the Role of Dentists in the Diagnosis and Treatment of Obstructive Sleep Apnea
}

Richard A. Bonato, Ph.D.

Chief Executive Officer, BRAEBON Medical Corporation, Ottawa Canada

I have recently read the article by Quan \& SchmidtNowara ${ }^{1}$ entitled "The role of dentists in the diagnosis and treatment of obstructive sleep apnea" as well as a followup editorial by Masse ${ }^{2}$, "On the Role of Dentists in the Diagnosis and Treatment of Obstructive Sleep Apnea." Having been involved in sleep research, sleep medicine and dental sleep medicine for over 30 years in academia, hospital clinical settings, and industry, I take issue with one particular statement made by Quan \& Schmidt. Specifically, I disagree with their position that dentists should be "strongly discouraged" from dispensing home sleep apnea testing (HSAT) devices for the purpose of using the interim results for oral appliance therapy (OAT) titration.

During the past few decades, we have observed tremendous change in the field of sleep medicine. Paper polysomnography became paperless and Continuous Positive Airway Pressure (CPAP) has continuously improved with smaller machines, warm pass-over humidification, better masks, and routine compliance tracking. All of this contributed to an exponential growth in the number of sleep laboratories during the 1990s. Dental sleep medicine has also evolved as can be observed from monobloc designs giving way to duobloc, traditional acrylic to CAD/CAM options, availability of digital impressions, and the creation of affordable objective OAT compliance measurement. Change is the perpetual constant. In 2008, change in the form of HSAT approval by the US Centers for Medicare \& Medicaid Services, and later by private insurance payers, came to the United States. From a technology perspective this change was more or less trivial because the data was simply stored inside a wearable device rather than sent down the laboratory hallway to a computer. However, for patients this was a substantial change because people could sleep in the comfort and convenience of their own beds rather than being forced to sleep in strange laboratory environments. In other words, the patient as the data unit remained the same but the data storage location was different. However, data is data and all the controversy regarding laboratory vs. HSAT resulted in naught. For example, Rosen et al. ${ }^{3}$ concluded that home-based diagnosis and treatment was not inferior to a laboratory approach in terms of patient acceptance, adherence, time to treatment, and functional improvements.

The pace of technological change has continued in the recent past. During the past 10 years, smartphones have become ubiquitous and most everyone is walking around with powerful pocket computers. Personal health, fitness, and sleep and snoring apps as well as ancillary products are bountiful. Many people track their sleep / activity for personal knowledge. This trend will only continue and personal health data access will become more accessible. Though not medical devices per se, smartphones offer instant communication, data collection and storage. Similarly, telemedicine offers instant remote access and is helping revolutionize sleep apnea diagnosis and treatment. Rural or remote patients need no longer suffer disadvantage because of their physical location or lack of access to a specialist physician. A physician may conduct a patient exam, order an HSAT, have it validated by a sleep technologist, review the raw data, make a diagnosis and write a prescription via telemedicine all from the comfort of the physician's home office. There is a role for dental sleep medicine within this modern telemedicine process.

The typical dental office has a patient recall once every six months and the public has been ingrained to "see your dentist twice a year." The average middle-aged male will visit their dentist more frequently than their family physician. Recent research suggests the global prevalence of sleep apnea approaches one billion people which represents epidemic levels ${ }^{4}$ and an American Academy of Sleep Medicine report estimates the annual cost of sleep apnea to be $\$ 150$ billion per annum to the US economy alone ${ }^{5}$. Therefore, there is an urgent need for much more sleep apnea evaluation, not less, and adopting modern technology to encourage collaboration between allied healthcare practitioners is the most comprehensive and logical means to address this dilemma. The appropriate use of modern HSAT technology, for example, in suitable patients without significant comorbidities, may be used by dentists to help identify sleep bruxism prior to initiating prosthodontic reconstruction. In addition, a dentist who suspects airway issues following a comprehensive clinical 
intake, performs an HSAT, and subsequently refers the patient with accompanying HSAT data to a local otolaryngologist is the perfect model of the type of cooperation which is needed. A dentist is trained to read an $\mathrm{x}$-ray but dentists do not diagnose cancer if they observe something unusual in a CBCT scan. A concern in a scan results in a referral to a radiologist. Similarly, a dentist should use an HSAT for guidance during the OAT titration period and subsequently return the patient to the sleep physician along with patient HSAT data to close the communication loop after optimal titration is observed. HSAT inexpensively extends healthcare into our communities beyond smartphone apps and the combination with telemedicine efficiently permits dentists and physicians to evaluate airway issues together. Dentists are experts in upper airway anatomy, physiology and musculature, and are highly educated regarding the tongue, swallowing, the temporomandibular joint, and recognition of oral cancer among other conditions. When a dentist looks inside a mouth they are looking for much more than simply cavities and periodontal decay. Scalloping of the tongue, abfractions, TMD, a narrow airway, large neck circumference, and, of course, snoring, are all possible indicators of obstructive sleep apnea. ${ }^{6,7}$ For these reasons, modern dentistry is a valuable component to address the enormous sleep apnea public health issue.

Everyone agrees sleep apnea is a medical disorder which requires a diagnosis from a qualified sleep physician followed by a prescription for a sleep apnea oral appliance before any dentist begins OAT. However, the issue of airway is part of routine day to day dentistry whether a practitioner is actively involved in patient airway management or blissfully ignorant. Anytime a dentist inserts an appliance in the mouth they may impact tongue space and deleteriously affect airway. Gagnon et al. ${ }^{8}$ reported that 5 of 10 patients had their sleep apnea worsen when using a single arch maxillary night guard for treating sleep bruxism. To ignore the association between tongue space, airway, and dentistry when using sleep bruxism, sleep apnea, or any other removable intraoral appliance is to invite malpractice. To suggest that dentists are somehow incapable of evaluating successful OAT titration using objective data and should be relegated to the mere technical issues surrounding oral appliance fabrication and fitting undermines the field of dental sleep medicine. All good clinicians, medical, dental or otherwise, should compare baseline data against follow-up (treatment) data and work collaboratively and cooperatively together to share the data for the best overall patient care. Modern $21^{\text {st }}$ Century technology is available today to eliminate data-sharing barriers and empower allied healthcare practitioners to effectively manage patients together.

\section{CITATION}

Bonato, RA. Removing barriers: On the role of dentists in the diagnosis and treatment of obstructive sleep apnea. Journal of Dental Sleep Medicine. 2018;5(3):104-105.

\section{REFERENCES}

1. Quan SF, Schmidt-Nowara W. The role of dentists in the diagnosis and treatment of obstructive sleep apnea: consensus and controversy. Journal of Clinical Sleep Med. 2017;13(10):1117-1119.

2. Masse JF. On the role of dentists in the diagnosis and treatment of obstructive sleep apnea: controversy and controversy. Journal of Dental Sleep Medicine. 2018; 5(2): 29-30.

3. Rosen, C., Auckley, D., Benca, R., Foldvary-Schaefer, N., Iber, C., Kapur, V., Rueschman, M., Zee, P., Redline, S. A Multisite Randomized Trial of Portable Sleep Studies and Positive Airway Pressure Autotitration Versus Laboratory-Based Polysomnography for the Diagnosis and Treatment of Obstructive Sleep Apnea: The HomePAP Study. Sleep. 2012; 35(6): 757-767.

4. Benjafield, A., Valentine, K., Ayas, N., Eastwood, P., Heinzer, R., Ip, M., Patel, S., Peppard, P., Sinha, S., Tufik, S., Nunez, C., Malhotra, A. Global Prevalence of Obstructive Sleep Apnea in Adults: Estimation Using Currently Available Data. San Diego: American Thoracic Society Annual Meeting. 2018, A3962-A3962.

5. American Academy of Sleep Medicine. Hidden Health Crisis Costing America Billions: Underdiagnosing and Undertreating Obstructive Sleep Apnea Draining Healthcare System. 2016.

6. Weiss, T., Atanasov, S., Calhoun, KH. The association of tongue scalloping with obstructive sleep apnea and related sleep pathology. Otolaryngol Head Neck Surg. 2005; 133(6): 966-71.

7. Sanders, A., Essick, G., Fillingim, R., Knott, C., Ohrbach, R., Greenspan, J., Diatchenko, L., Maixner, W., Dubner, R., Bair, E., Miller, V., Slade, G. Sleep Apnea Symptoms and Risk of Temporomandibular Disorder: OPPERA Cohort. Journal of Dental Research. 2013; 92(7): S70-S77.

8. Gagnon, Y., Mayer, P., Morisson, F., Rompré, PH., Lavigne GJ. Aggravation of respiratory disturbances by the use of an occlusal splint in apneic patients: a pilot study. Int $J$ Prosthodont. 2004; 17(4):447-53.

\section{SUBMISSION AND CORRESPONDENCE INFORMATION}

\author{
Submitted in final revised form June 29, 2018 \\ Accepted for publication June 29, 2018
}

Address correspondence to: Richard A. Bonato, Ph.D., Chief Executive Officer, BRAEBON Medical Corporation, Suite 1, 100 Schneider Road, Kanata, Ontario, Canada, K2K 1Y2; Email: rick@braebon.com

\section{DISCLOSURE STATEMENT}

The author is an executive of BRAEBON Medical Corporation. 